\title{
SUPPORT TO THE NAVIGATION OF A ROBOT USING ARTIFICIAL NEURAL NETWORKS
}

\section{CÉSAR A. PERDOMO CH., LEONARDO PLAZAS-NOSSA \& JULIÁN R. CAMARGO L.}

Engineering Faculty, Universidad Distrital Francisco José de Caldas, Bogotá, Colombia

\begin{abstract}
This document proposes the modeling of a neural network for the classification of the movement of a robot from ultrasound sensors that provide information about the environment of the same. Three models with a different number of neurons in the hidden layer are tested using the conjugated gradient learning algorithm. Ten training sessions are performed with each of these models, giving the best result of each model.

KEYWORDS: Backpropagation, Classification, Control, Navigation, Neural Networks
\end{abstract}

Received: Oct 04, 2020; Accepted: Oct 24, 2020; Published: Nov 23, 2020; Paper Id.: IJMPERDOCT202063

\section{INTRODUCTION}

The use of artificial neural networks to find alternative solutions to complex problems is becoming more frequent in different areas of knowledge. That is why it is not rare to find the use of this paradigm of computational intelligence in areas such as medicine, meteorology, banking, etc.

One of the most frequent problems that we can solve with ANNs is those that refer to classification, wherefrom given characteristics the model must present correspondence to one of the categorical variables that are presented as valid. However, one of the main problems with the abundance of data and the mass use of increasingly large storage systems is the extraction of characteristics that have causality with the model output and that do not present redundancy for the development of the model.

For this reason, it is very important to carry out a strict and careful exploratory analysis of the data, where the different statistical and graphical analyses are used to find the number of variables in the model appropriate to the complexity of the problem, in this way, being a little more coherent with the dimensionality of the models without sacrificing the accuracy of the same.

The purpose of this article is the design of a controller of the movement of a robot considering the 4 characteristics calculated and defined by the exploratory analysis of the data starting from the reading of the sensors that are in its structure and to establish the exit of movement adapted for those readings.

\section{MATERIALS}

\subsection{Classification models with neural networks}

The use of neural networks for classification by imitating the capacity of the human brain to learn from experience to perform better action in future situations. The above is the principle of supervised learning. Each artificial neuron 
offers a processing capacity that together with an activation function allows us to obtain an output that is taken into account in the following layers, this occurs in multilayer models such as the Backpropagation network. A 3-layer backpropagation network is shown in Figure 1.

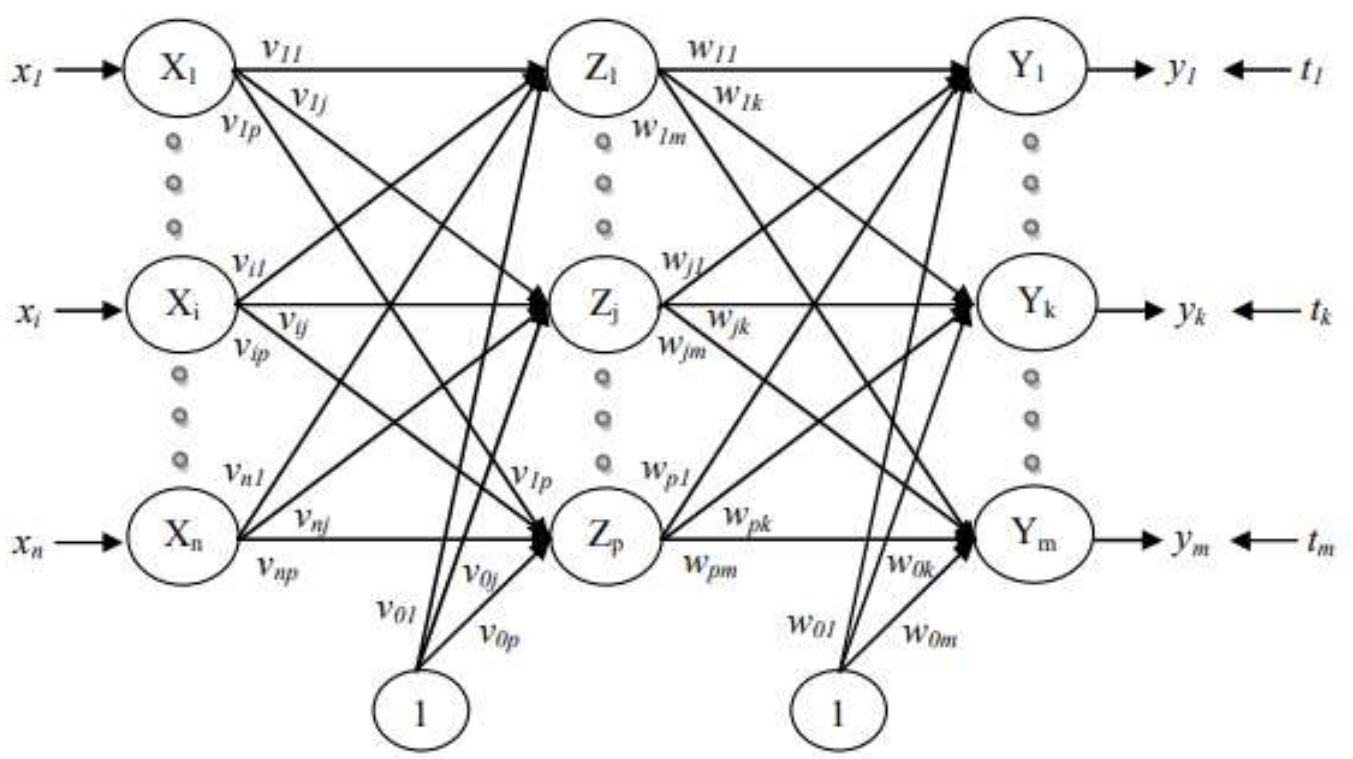

Figure 1: Three-layer backpropagation neural network [1]

The four phases of the training consist of initialization of the model weights, forward propagation, backward propagation of errors and updating of model weights. The updating of weights aims at each iteration to reduce the error to a minimum.

The methodology for the development of the model is initially to load the data set, four inputs make up the model inputs and four classes of output. The model is trained with the Scaled conjugate gradient backpropagation algorithm. The training data is partitioned $80 \%, 10 \%$ for validation and the remaining $10 \%$ for testing.

\subsubsection{Scaled Conjugate Gradient (Trainscg)}

The Scaled Conjugate Gradient (SCG) algorithm was modeled to circumvent the search for lines that require a considerable amount of time. This algorithm combines the model-trust region approach used in the Levenberg-Marquardt algorithm, with the conjugate gradient approach. [2]

\subsection{Dataset}

The SCITOS G5 robot (Figure 2) contains ultrasound sensors located around it and thus be able to locate the obstacles in the room [3]. It forms a first dataset that contains the 24 sensors with $15^{\circ}$ separation and this achieves surround the entire robot. The numbering of the sensors is done from the front following the direction of the hands of the clock [4].

The second set of data is calculated from the first allowing this way to train models with a lower dimensionality because the number of inputs decreases from 24 to 4 . These four data are calculated from the minimum reading value of the sensor in a range of $60^{\circ}$ in each of the four main positions of the robot: Front, left, right and back [5]. 


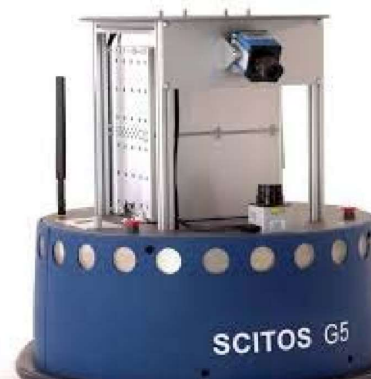

Figure 2: SCITOS G5 mobile robot

These actions to take the robot are divided into the following 4 classes: moving forward, a slight turn to the right, a sharp turn to the right and a slight turn to the left.

The records were captured at a frequency of 9 samples per second [6]. For a total of 5456 samples.

\section{METHODS}

For the training of the model proposed in Figure 3. Where the number of neurons in the hidden layer can be modified. The training algorithm for this model was the Scaled Conjugate Gradient (Trainscg) [7] using MATLAB® 2019b software.

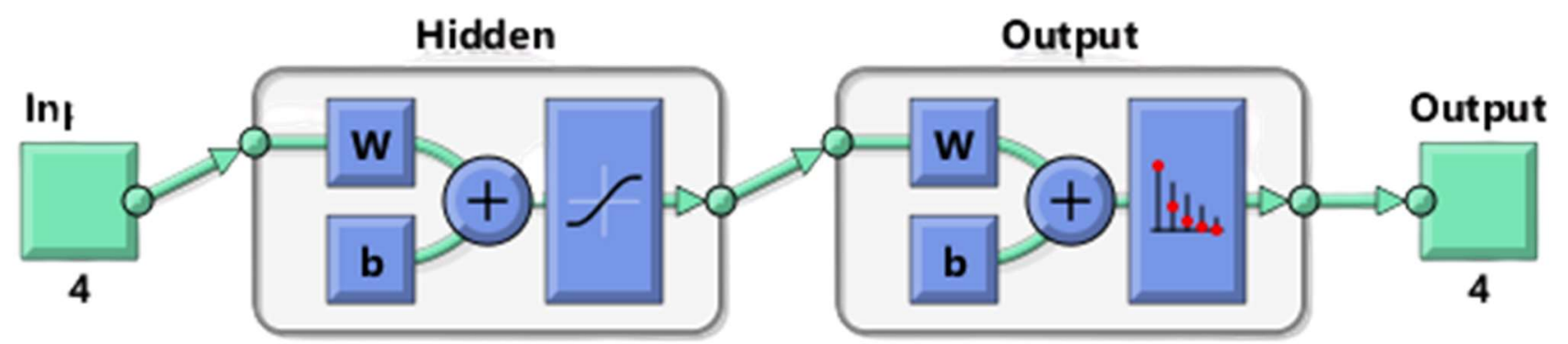

Figure 3: Proposed model for classification

The adjustment of the model is going to be done based on the Backpropagation model so the activation functions of all the layers must be derivable [8]. In the case of the hidden layer, the sigmoid activation function shown in Figure 4 was used.

$$
S(x)=\frac{1}{1+e^{-x}}
$$

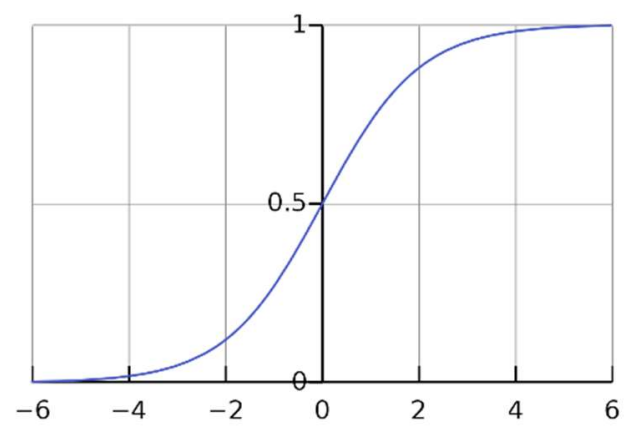

Figure 4: Sigmoid activation function used in the hidden layer 
The training of the model is carried out with 4,364 records that correspond to $80 \%$ of the total records $(5,456)$. For the validation and testing of the model, 546 records are used, which are randomly separated from the dataset to guarantee that each subset obtains characteristics similar to the original dataset.

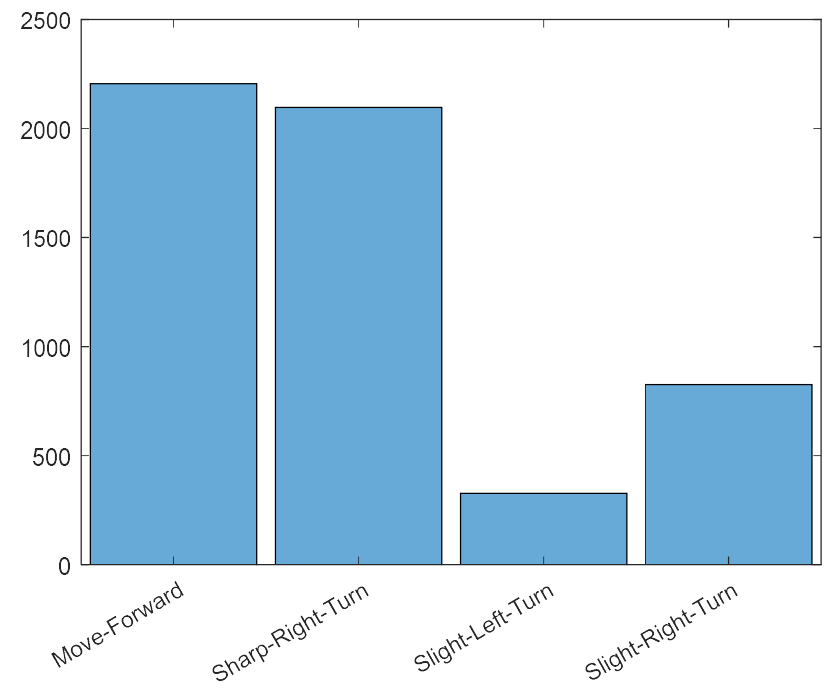

Figure 5: Histogram of the dataset output variable

The model entries are composed of values of which the change of the class variable by numerical variable was performed as follows (Table 1):

Table 1: Change to numerical output variable type for training

\begin{tabular}{|l|l|}
\hline \multicolumn{1}{|c|}{ Class } & Numerical value \\
\hline Move-Forward & 1 \\
\hline Sharp-Right-Turn & 2 \\
\hline Slight-Left-Turn & 3 \\
\hline Slight-Right-Turn & 4 \\
\hline
\end{tabular}

\section{RESULTS}

Three models with different numbers of neurons in the hidden layer were proposed in such a way that the difference between the effectiveness of each one of the models for the three data sets (training, validation and test) can be evidenced [9], [10].

The confusion matrices of each of these models are shown below and in Table 2 the summary results of the three models for the test subset can be visualized.

Table 2: Summary results of the three models

\begin{tabular}{|c|c|c|c|c|}
\hline \multirow{2}{*}{\multicolumn{2}{|c|}{ Class }} & \multicolumn{3}{|c|}{ Number of hidden neurons } \\
\hline & & \multirow{2}{*}{$\begin{array}{c}10 \text { Neurons } \\
96.0 \%\end{array}$} & \multirow{2}{*}{ 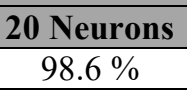 } & \multirow{2}{*}{$\begin{array}{c}30 \text { Neurons } \\
99.5 \% \\
\end{array}$} \\
\hline 1 & Move-Forward & & & \\
\hline 2 & Sharp-Rigth-Turn & $98.0 \%$ & $98.6 \%$ & $97.7 \%$ \\
\hline 3 & Slight-Left-Turn & $100 \%$ & $100 \%$ & $100 \%$ \\
\hline 4 & Slight-Rigth-Turn & $84.8 \%$ & $94.0 \%$ & $94.6 \%$ \\
\hline & Total accuracy & $94.1 \%$ & $97.8 \%$ & $98.0 \%$ \\
\hline
\end{tabular}



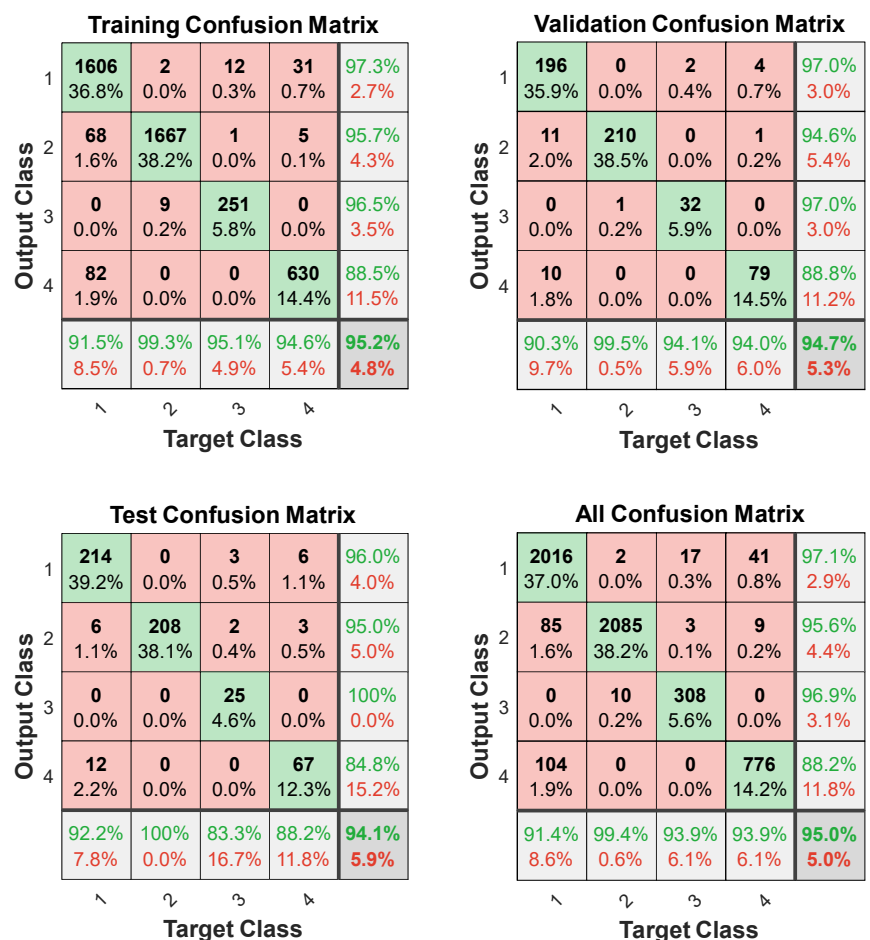

Figure 6: Model 1 confusion matrix (10 neurons in the hidden layer)
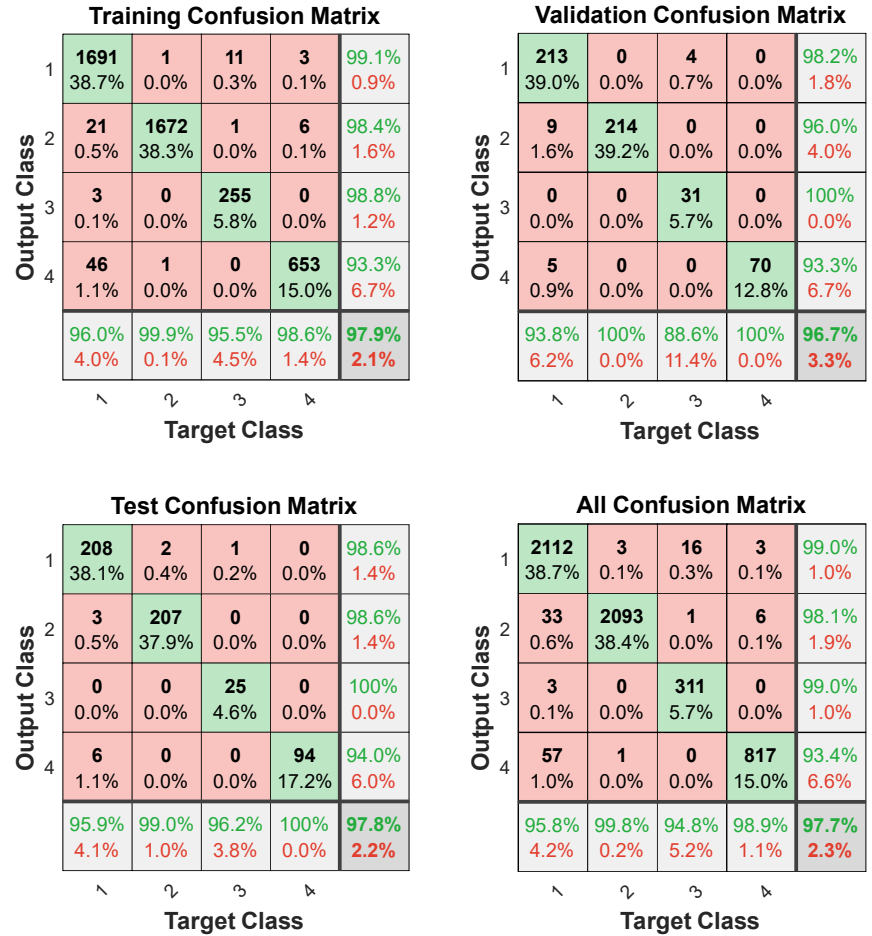

Figure 7: Model 2 confusion matrix (20 neurons in the hidden layer) 

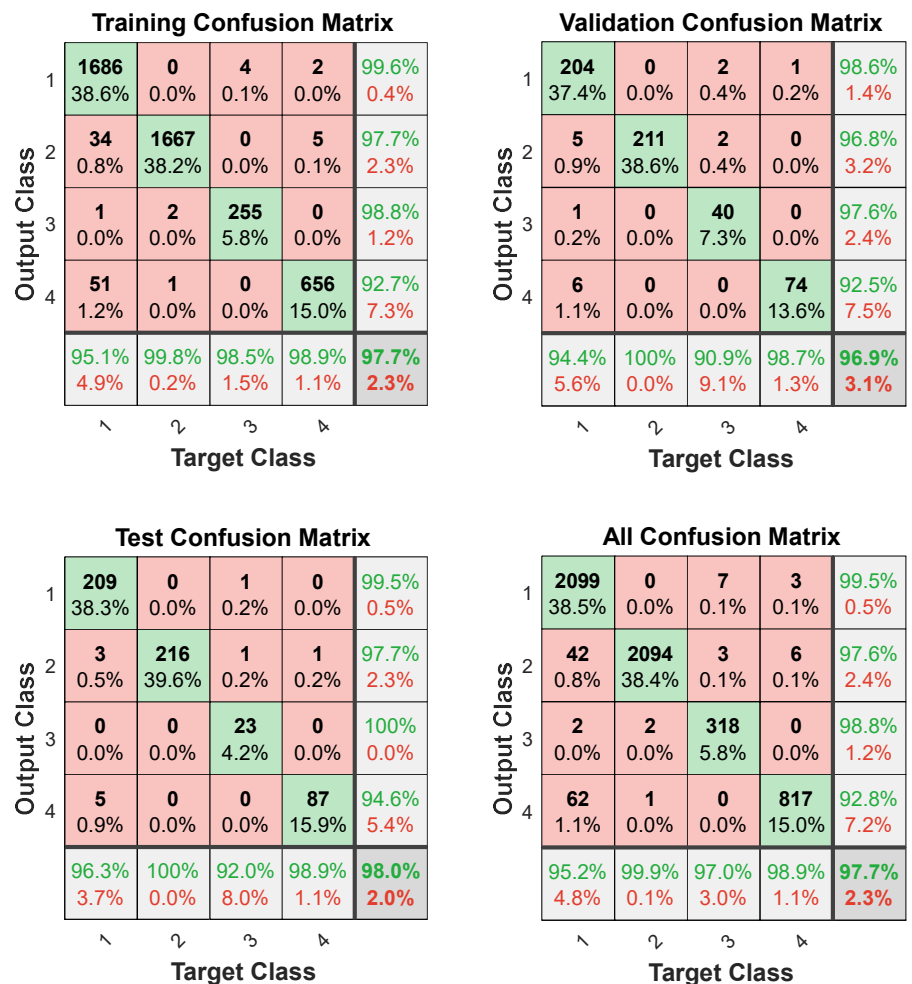

Figure 8: Model 3 confusion matrix (30 neurons in the hidden layer)

\section{CONCLUSIONS}

The use of neuronal networks for classification of the robot's movement presents good results independent of the number of neurons in the hidden layer of the trained models, presenting a minimum accuracy of the model of $94 \%$ for the test subset.

Although the dataset is unbalanced, there are no significant differences in the accuracy of the model by class, presenting better results in the Slight-Left-Turn class that presents a lower number of records.

In model 1, the total effectiveness of the classifier for class 3 in the test subset is presented, allowing evidence that the model is not over-adjusted because it presents a better precision in comparison with the behavior of the same class in the training subset.

Model 3 shows a better overall behavior because it presents a better accuracy with the Move-Forward class which has a higher number of records and thus significantly improving the overall accuracy of the model.

\section{ACKNOWLEDGMENTS}

The authors would like to thank the Universidad Distrital Francisco José de Caldas and the LASER research group that supported the development and testing of the project.

\section{REFERENCES}

1. Rahim, N. A., Paulraj, M. P., Adom, A. H., \& Sundararaj, S. (2010, May). Moving vehicle noise classification using backpropagation algorithm. In 2010 6th International Colloquium on Signal Processing \& Its Applications (pp. 1-6). IEEE. 
2. Sivasankari, K., Thanushkodi, K., \& Kalaivanan, K. (2013, July). Automated seizure detection using multilayer feed-forward network trained using scaled conjugate gradient method. In 2013 International Conference on Current Trends in Engineering and Technology (ICCTET) (pp. 195-198). IEEE.

3. Wall-Following Robot Navigation Data Data Set, Machine Learning Repository, University of California, Irvine (UCI). Retrieved from: https://archive.ics.uci.edu/ml/datasets/Wall-Following+Robot+Navigation+Data

4. Christian Martin, SCITOS G5 - A mobile platform for research and industrial, MetraLabs GmBH, Mobile Robotics. Retrieved from: http://download.ros.org/data/Events/CoTeSys-ROSSchool/metralabs.pdf

5. Dash, Tirtharaj, et al. (2014). Neural network approach to control wall-following robot navigation. Advanced Communication Control and Computing Technologies (ICACCCT), 2014 International Conference on. IEEE, 2014.

6. Freire, Ananda L., et al. (2009). Short-term memory mechanisms in neural network learning of robot navigation tasks: A case study. Robotics Symposium (LARS), 2009 6th Latin American. IEEE, 2009.

7. Møller, M. F. (1993). A scaled conjugate gradient algorithm for fast supervised learning. Neural networks, 6(4), 525-533.

8. Johansson, E. M., Dowla, F. U., \& Goodman, D. M. (1991). Backpropagation learning for multilayer feed-forward neural networks using the conjugate gradient method. International Journal of Neural Systems, 2(04), 291-301.

9. Errica, F., Podda, M., Bacciu, D., \& Micheli, A. (2019). A fair comparison of graph neural networks for graph classification. arXiv preprint arXiv:1912.09893.

10. More, A. (2016). Survey of resampling techniques for improving classification performance in unbalanced datasets. arXiv preprint arXiv:1608.06048 

